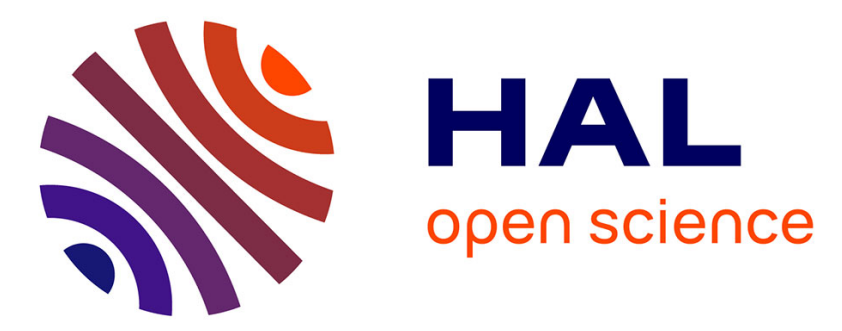

\title{
Asymptotic expressions for the nearest and furthest dislocations in a pile-up against a grain boundary
}

Cameron Luke Hall

\section{To cite this version:}

Cameron Luke Hall. Asymptotic expressions for the nearest and furthest dislocations in a pile-up against a grain boundary. Philosophical Magazine, 2010, 90 (29), pp.3879. 10.1080/14786435.2010.499859 . hal-00608030

\section{HAL Id: hal-00608030 \\ https://hal.science/hal-00608030}

Submitted on 12 Jul 2011

HAL is a multi-disciplinary open access archive for the deposit and dissemination of scientific research documents, whether they are published or not. The documents may come from teaching and research institutions in France or abroad, or from public or private research centers.
L'archive ouverte pluridisciplinaire HAL, est destinée au dépôt et à la diffusion de documents scientifiques de niveau recherche, publiés ou non, émanant des établissements d'enseignement et de recherche français ou étrangers, des laboratoires publics ou privés. 


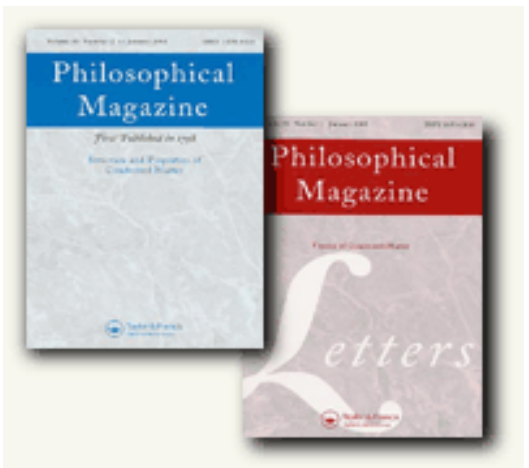

\section{Asymptotic expressions for the nearest and furthest dislocations in a pile-up against a grain boundary}

\begin{tabular}{|c|c|}
\hline Journal: & Philosophical Magazine \& Philosophical Magazine Letters \\
\hline Manuscript ID: & TPHM-10-Jan-0005.R2 \\
\hline Journal Selection: & Philosophical Magazine \\
\hline $\begin{array}{r}\text { Date Submitted by the } \\
\text { Author: }\end{array}$ & 24-May-2010 \\
\hline Complete List of Authors: & Hall, Cameron; Oxford University, OCCAM, Mathematical Institute \\
\hline Keywords: & dislocation theory, dislocation interactions, grain boundaries \\
\hline Keywords (user supplied): & asymptotic analysis \\
\hline \multicolumn{2}{|c|}{$\begin{array}{l}\text { Note: The following files were submitted by the author for peer review, but cannot be converted } \\
\text { to PDF. You must view these files (e.g. movies) online. }\end{array}$} \\
\hline Resubmission ArmstrongNot & zip \\
\hline
\end{tabular}

\section{S ScholaroNE \\ Manuscript Central}




\title{
RESEARCH ARTICLE
}

\section{Asymptotic expressions for the nearest and furthest dislocations in a pile-up against a grain boundary}

\author{
Cameron L. Hall \\ (Received 00 Month 200x; final version received 00 Month 200x)
}

\begin{abstract}
In 1965, Armstrong and Head (Acta Metall. 13(7):759-764, 1965) explored the problem of a pile-up of screw dislocations against a grain boundary. They used numerical methods to determine the positions of the dislocations in the pile-up and they were able to fit approximate formulae for the locations of the first and last dislocations. These formulae were used to gain insights into the Hall-Petch relationship. More recently, Voskoboinikov et al. (Phil. Mag. Lett. 87(9):669-676, 2007) used asymptotic techniques to study the equivalent problem of a pile-up of a large number of screw dislocations against a bimetallic interface.

In this paper, we extend the work of Voskoboinikov et al. to construct systematic asymptotic expressions for the formulae proposed by Armstrong and Head. The further extension of these techniques to more general pile-ups is also outlined. As a result of this work, we show that a pile-up against a grain boundary can become equivalent to a pile-up against a locked dislocation in the case where the mismatch across the boundary is small.
\end{abstract}

\section{Introduction}

In their analysis of brittle fracture of polycrystalline materials, Armstrong and Head [1] investigated a pile-up of screw dislocations against a grain boundary. Specifically, they considered the case of an elastically anisotropic material where the crystal orientation changes across a grain boundary. This situation can be dealt with effectively by using the method of images, in which each real dislocation generates a stress field corresponding to a virtual dislocation situated at the image point opposite the grain boundary. The strength of the image dislocation is an explicitly known quantity that depends on the degree of elastic anisotropy in the crystal and on the misorientation across the boundary. Analogous pile-ups of screw dislocations against a bimetallic interface have recently been investigated by Voskoboinikiov et al. [8] in the asymptotic limit as the number of dislocations tends to infinity.

In [1], numerical methods were used to solve the equations of equilibrium for pile-ups of up to 64 dislocations. Based on these results, heuristic formulae were given for the positions of the first and last dislocations in the pile-up. In all cases described, it was found that the dimensionless position of the first dislocation could be simply expressed in terms of the number of real dislocations in the pile-up and the comparative strength of an image dislocation relative to a real dislocation. In this paper, the results of Voskoboinikov et al. [8] are used to develop asymptotic expressions for the positions of the first and last dislocations in a pile-up of screw dislocations against a grain boundary. These expressions give a good justification for the formulae obtained in [1], but we also find some important differences.

Armstrong and Head [1] considered the implications of their formulae for predicting brittle fracture of a polycrystalline material. By making appropriate assumptions, they recovered an expression for the yield stress that resembles the Hall-Petch criterion. We also consider this problem and obtain equivalent asymp-

ISSN: $1478-6435$ print/ISSN 1478-6443 online

(C) 200x Taylor \& Francis

DOI: $10.1080 / 1478643$ YYxxxxxxxx

http://www.informaworld.com 


\section{Pile-up against a grain boundary}

Armstrong and Head [1] considered both pile-ups against a grain boundary and pile-ups against a grain boundary and a locked dislocation. The former situation is identical to the problem investigated by Voskoboinikov et al. [8] and we analyse it first.

Consider a pile-up of $n$ screw dislocations, each with Burgers vector $\boldsymbol{b}=b \boldsymbol{k}$, in an infinite material containing a grain boundary at the plane $x=0$. The dislocations are taken to be parallel with the $z$-axis and are located in the plane $y=0$ at positions $x=x_{i}>0, i=1, \ldots, n$. The pile-up is held $\ll i n \gg$ place by a constant applied $y-z$ shear stress of magnitude $\tau$. The ratio of the strength of an image dislocation compared to a real dislocation is represented by $\lambda$; Armstrong and Head [1] observe that $|\lambda|<0.3$ for both iron and copper. by

With this notation, the equations for dislocation equilibrium for $\lambda>0$ are given

$$
\frac{\mu}{2 \pi} \sum_{j=1, j \neq i}^{n} \frac{1}{x_{i}-x_{j}}+\frac{\mu \lambda}{2 \pi} \sum_{j=1}^{n} \frac{1}{x_{i}+x_{j}}=\tau, \quad i=1,2, \ldots, n
$$

where $\mu$ is the equivalent shear modulus obtained from the anisotropic elasticity tensor [1]. Nondimensionalising space with respect to $\frac{\mu b}{2 \pi \tau}$, this leads to the following dimensionless system:

$$
\sum_{j=1, j \neq i}^{n} \frac{1}{x_{i}-x_{j}}+\lambda \sum_{j=1}^{n} \frac{1}{x_{i}+x_{j}}=1, \quad i=1,2, \ldots, n .
$$

When $\lambda<0$, equation (1) cannot be used to find $x_{1}$ because the attraction from the image dislocations would make the first real dislocation very close to the grain boundary. This implies the situation of a pile-up against a lock and a grain boundary, which we discuss in Section 3 . 


\section{ay 24,2010 \\ Philosophical Magazine \& Philosophical Magazine Letters}

\subsection{Position of the furthest dislocation}

As described by Voskoboinikov et al. [8], there are three spatial scalings to consider for (1). In the region where $x$ is sufficiently far from the boundary layers near $x=0$ and $x=x_{n}$, the spatial variable can be rescaled by introducing $x=n \xi$ to recover a singular integral equation for the leading-order dislocation density, $\rho_{0}(\xi)$. This integral equation can be solved using the Wiener-Hopf method, enabling us to find both $\rho_{0}(\xi)$ and the leading-order scaled location of the last dislocation, $\xi_{0}^{*}$. From $\ll[5] \gg$, these are given by

$$
\rho_{0}(\xi)=\frac{1}{\pi \sin (\pi a / 2)} \sinh \left(a \cosh ^{-1} \frac{\xi_{0}^{*}}{\xi}\right)
$$

and

$$
\xi_{0}^{*}=\frac{\sin (\pi a)}{a},
$$

where

$$
a=\frac{\cos ^{-1} \lambda}{\pi}
$$

Greater accuracy in the position of the furthest dislocation can be obtained by considering the boundary layer near $x=x_{n}$. Equation (2) indicates that $\rho_{0}(\xi) \sim$ $\frac{1}{\pi} \sqrt{c_{0}\left(\xi_{0}^{*}-\xi\right)}$ near $\xi_{0}^{*}$, where

$$
c_{0}=\frac{2 a^{3}}{\sin ^{2}(\pi a / 2) \sin (\pi a)} .
$$

Following the procedures in [9], we find that

$$
\xi_{n}=\xi_{0}^{*}+q c_{0}^{-\frac{1}{3}} n^{-\frac{2}{3}}+\mathcal{O}\left(n^{-1}\right)
$$

where $q$ is the first root of the Airy function, $\operatorname{Ai}(x)$.

Taking Taylor series around $\lambda=0$, this yields

$$
\xi_{n}=2\left(1+\frac{2}{\pi} \lambda\right)+2^{\frac{1}{3}} q n^{-\frac{2}{3}}\left(1+\left(\frac{2}{\pi}-\frac{1}{3}\right) \lambda\right)+\mathcal{O}\left(n^{-1}, \lambda^{2}\right) .
$$

Ignoring the $n^{-\frac{2}{3}}$ term would give the simpler expression

$$
\xi_{n}=2\left(1+\frac{2}{\pi} \lambda\right)+\mathcal{O}\left(n^{-\frac{2}{3}}, \lambda^{2}\right) .
$$

Using (5), we find that

$$
\begin{aligned}
x_{n} & =2 n\left(1+\frac{2}{\pi} \lambda\right)+2^{\frac{1}{3}} q n^{\frac{1}{3}}\left(1+\left(\frac{2}{\pi}-\frac{1}{3}\right) \lambda\right)+\mathcal{O}\left(1, n \lambda^{2}\right) \\
& \approx 2 n(1+0.64 \lambda)-2.94 n^{\frac{1}{3}}(1+0.30 \lambda) .
\end{aligned}
$$




\section{Philosophical Magazine \& Philosophical Magazine Letters}

For comparison, the equivalent expression obtained by Armstrong and Head [1] from curve-fitting is

$$
x_{n} \approx 2 n(1+0.9 \lambda) .
$$

We have developed a Newton iterative scheme for solving (1), using a starting condition where each dislocation is only able to feel its nearest neighbours and the external stress. This gave the numerical results for $\xi_{n}$ shown in Figure 1 for a variety of different values of $n$ and $\lambda$, including the values used in [1]. Interestingly, equations (6) and (8) both give a fairly poor fit to the final dislocation positions obtained using our numeric scheme. However, including the first correction term associated with the boundary layer gives an expression for $\xi_{n}$ that $\ll$ agrees well with the numerical results $\gg$ over a wide range of $\lambda$ values and is even $\ll$ quite $\gg$ good when $n$ is not very large.

\subsection{Position of the nearest dislocation}

We now obtain an expression for $x_{1}$, the location of the first dislocation in the pileup. Since this dislocation will lie in a boundary layer region near $x=0$, it requires a different spatial scaling from the one described above. Following Voskoboinikov et al. [8], we introduce an inner scaling of the form $x=n^{-a /(1-a)} \eta$, where $a$ is defined as above. As shown in [8], the leading-order inner equation takes the form

$$
\sum_{j=1, i \neq j}^{\infty} \frac{1}{\eta_{i}-\eta_{j}}+\sum_{j=1}^{\infty} \frac{\lambda}{\eta_{i}+\eta_{j}}=0, \quad i=1,2, \ldots
$$




\section{ay 24,2010

subject to the matching condition

$$
\eta_{k} \rightarrow 2\left(\frac{k \pi(1-a) \sin (\pi a / 2)}{\left(\xi_{0}^{*}\right)^{a}}\right)^{1 /(1-a)}
$$

as $k \rightarrow \infty$.

In Voskoboinikov et al. [8], equation (9) is solved using numerical methods. However, it is possible to make further algebraic progress by exploiting the assumption that $\lambda$ is small. We thus let each $\eta_{i}$ be represented as an asymptotic power series in $\lambda$ :

$$
\eta_{i} \sim \eta_{i, 0}+\lambda \eta_{i, 1}+\ldots
$$

Collecting only the leading-order terms in equation (9), we find that

$$
\sum_{j=1, i \neq j}^{\infty} \frac{1}{\eta_{i, 0}-\eta_{j, 0}}=0, \quad i=1,2, \ldots
$$

Also, we note that $a \sim \frac{1}{2}+\mathcal{O}(\lambda)$ and $\xi_{0}^{*} \sim 2+\mathcal{O}(\lambda)$. Hence, the leading-order matching condition (10) becomes

$$
\eta_{k, 0} \sim \frac{k^{2} \pi^{2}}{8}
$$

as $k \rightarrow \infty$. However, (11) is clearly incorrect when $i=1$, as all of the terms on the left-hand side are negative. We attempt to resolve this problem by introducing a different scaling for just $\eta_{1}$. Specifically, we propose $\eta_{1}=\lambda \eta_{1,0}+o(\lambda)$ and thus we find that the leading-order equation for $i=1$ takes the form

$$
\frac{1}{2 \eta_{1,0}}-\sum_{j=2}^{\infty} \frac{1}{\eta_{j, 0}}=0
$$

while the other leading-order equations are given by

$$
\sum_{j=2, i \neq j}^{\infty} \frac{1}{\eta_{i, 0}-\eta_{j, 0}}+\frac{1}{\eta_{i, 0}}=0, \quad i=2,3, \ldots
$$

Importantly, we note that the system (14) is completely independent of $\eta_{1,0}$. Moreover, this system of equations is identical to the system described in Voskoboinikov et al. [9] for the inner problem of a pile-up of dislocations against a lock. Indeed, when $\lambda$ is small, we find that the dislocation $x_{1}$ is precisely equivalent to a locked dislocation to lowest order.

Unlike the system (9), (14) has the advantage that it can be solved exactly. Following Voskoboinikov et al. [9] and applying the matching condition (12), we find that

$$
\eta_{i, 0}=\frac{\left(\alpha_{i-1}\right)^{2}}{8}, \quad i=2,3, \ldots
$$

where $\alpha_{k}$ is the $k$ th positive zero of the Bessel function $J_{1}(x)$. 
Rearranging equation (13), this implies that

$$
\eta_{1,0}=\frac{1}{16}\left(\sum_{i=1}^{\infty} \frac{1}{\alpha_{i}^{2}}\right)^{-1}
$$

We can now exploit the fact that

$$
\sum_{k=1}^{\infty} \frac{1}{\left(j_{\nu, k}\right)^{2}}=\frac{1}{4(\nu+1)}
$$

where $j_{\nu, k}$ is the $k$ th zero of the Bessel function, $J_{\nu}(x)$, and $\nu \geq 0$ to show that

$$
\eta_{1,0}=\frac{1}{2}
$$

and hence,

$$
x_{1} \sim \frac{\lambda}{2 n^{1-\frac{4}{\pi} \lambda}} \approx \frac{\lambda}{2 n^{1-1.27 \lambda}} .
$$

For comparison, the equivalent expression obtained by Armstrong and Head [1] is

$$
x_{1} \approx \frac{\lambda}{2 n^{1-1.1 \lambda}}
$$

The discrepancy between (16) and (17) can be investigated by plotting values of $\log \left(\frac{2 x_{1}}{\lambda}\right) / \log n$ obtained using the numerical procedure described above against $\lambda$, as shown in Figure 2. From this, we clearly see that (16) gives a good approximation of these numerical results when $\lambda$ is sufficiently small, but that the relationship between $\log \left(\frac{2 x_{1}}{\lambda}\right) / \log n$ and $\lambda$ quickly deviates from linearity as $\lambda$ increases. Indeed, for suitably large values of $\lambda$, the Armstrong-Head formula (17) is a better approximation of the numerical results than the asymptotic formula (16).

\section{Pile-up against a lock and a grain boundary}

Armstrong and Head [1] also consider the situation where there is a locked dislocation at $x=0$ in addition to image dislocations associated with the grain boundary. In this case, the equations of equilibrium take the form

$$
\sum_{j=1, j \neq i}^{n} \frac{1}{x_{i}-x_{j}}+\lambda \sum_{j=1}^{n} \frac{1}{x_{i}+x_{j}}+\frac{1+\lambda}{x_{i}}=1, \quad i=1,2, \ldots, n
$$

The presence or absence of a locked dislocation at $x=0$ will not affect the equilibrium equations for the dislocation density in the outer region. Hence, we can repeat the methods of the previous section to find that

$$
x_{n} \sim 2 n\left(1+\frac{2}{\pi} \lambda\right)+2^{\frac{1}{3}} q n^{-\frac{2}{3}}\left(1+\left(\frac{2}{\pi}-\frac{1}{3}\right) \lambda\right) .
$$




\section{Page 7 of 12 \\ Philosophical Magazine \& Philosophical Magazine Letters}

1

3

4

5

7

8

9

10

11

12

13

14

15

16

17

18

19

20

21

22

23

24

25

26

27

28

29

30

31

32

33

34

35

36

37

38

39

40

41

42

43

44

45

46

47

48

49

50

51

52

53

54

55

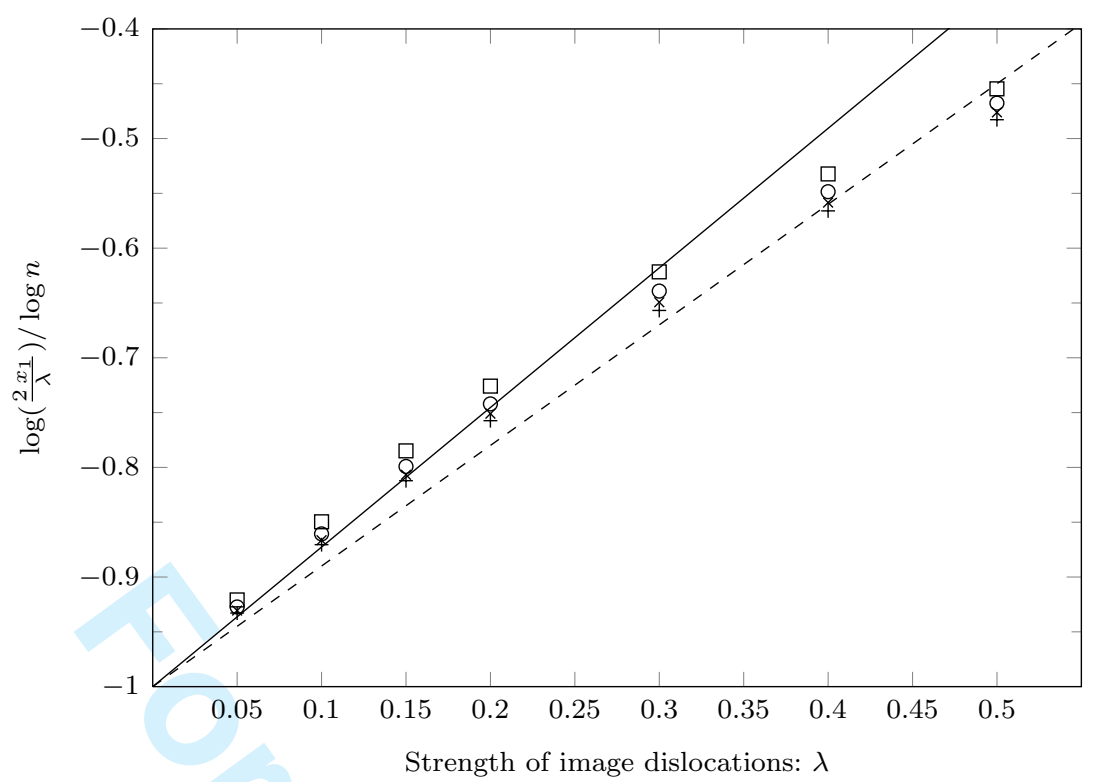

Figure 2. Numerically obtained results for $\log \left(\frac{2 x_{1}}{\lambda}\right) / \log n$ using a variety of values of $n$ and $\lambda$. Results for $n=4$ are shown as $\square$, results for $n=16$ are shown as $\bigcirc$, results for $n=64$ are shown as $\times$ and results for $n=400$ are shown as + . The asymptotic approximation $\log \left(\frac{2 x_{1}}{\lambda}\right) / \log n=-1+\frac{4}{\pi} \lambda$ is shown as a continuous line while the Armstrong-Head formula $\log \left(\frac{2 x_{1}}{\lambda}\right) / \log n=-1+1.1 \lambda$ is shown as a dashed line.

This is in general agreement with Armstrong and Head [1], who found that the position of the $n$th dislocation is not noticeably affected by the addition of a locked dislocation at $x=0$. They reached this conclusion by comparing numerical results for the two cases and finding that they converged as $n$ increased.

However, the locked dislocation will affect the solution in the inner region. In order to match with the unchanged outer region, we find that the same scalings apply as for the simple pile-up against a grain boundary. However, the inner system now takes the form

$$
\sum_{j=1, i \neq j}^{\infty} \frac{1}{\eta_{i}-\eta_{j}}+\sum_{j=1}^{\infty} \frac{\lambda}{\eta_{i}+\eta_{j}}+\frac{1+\lambda}{\eta_{i}}=0, \quad i=1,2, \ldots
$$

As before, we consider the case where $\lambda$ is small and we propose solutions for $\eta_{i}$ as asymptotic power series in $\lambda$. This leads us to recover the leading-order system

$$
\sum_{j=1, i \neq j}^{\infty} \frac{1}{\eta_{i, 0}-\eta_{j, 0}}+\frac{1}{\eta_{i, 0}}=0, \quad i=1,2, \ldots
$$

Because of the repulsion from the lock, the $i=1$ equation is no longer special and we can directly use the solution for a pile-up against a lock given by Voskoboinikov et al. [9]. Using this method, we find that

$$
\eta_{1,0}=\frac{\alpha_{1}^{2}}{8}
$$

where $\alpha_{1}$ is the first positive zero of the Bessel function $J_{1}(x)$. Thus,

$$
x_{1} \sim \frac{\alpha_{1}^{2}}{8 n^{1-\frac{4}{\pi} \lambda}} \approx \frac{1.84}{n^{1-1.27 \lambda}}
$$


Armstrong and Head [1] observed that plots of $\log x_{1}$ against $\log n$ based on the numerical solution of (18) yielded curves that were almost parallel to equivalent plots of $\log x_{1}$ against $\log n$ from the numerical solution of (1). This agrees with the observation that introducing a locked dislocation does not change the scaling used in the boundary layer.

Armstrong and Head [1] also found that, when extrapolated to $n=1$, the plots of $\log x_{1}$ against $\log n$ based on (18) converged to the same point for all values of $\lambda$. Thus, they obtained an expression for $x_{1}$ of the form

$$
x_{1} \approx \frac{1.9}{n^{1-1.1 \lambda}}
$$

Finally, they noted that this agrees well with the approximate result,

$$
x_{1} \sim \frac{\alpha_{1}^{2}}{8 n} \approx \frac{1.84}{n}
$$

obtained by Eshelby et al. [3] for the well-studied problem of a pile-up against a locked dislocation (i.e. with $\lambda=0$ ).

\section{More general pile-ups}

One limitation of [1] is that only pile-ups of screw dislocations are considered. For edge dislocations, more complicated expressions for the image forces are required. Additionally, it is possible to encounter pile-ups of edge dislocations where the plane containing the dislocations is not perpendicular to the plane of the grain boundary. In the simple case of a bimetallic interface (i.e. an interface between two elastically isotropic materials with different elastic constants), the full image stress is given by Head [4] and perpendicular pile-ups of edge dislocations have been analysed by Kuang and Mura [5] and by Voskoboinikov et al. [7].

In the more general case where there is a planar boundary between two different elastically anisotropic materials, Tucker [6] gives an expression for the interaction forces between a pair of straight dislocations. Except in the case of a dramatic change in material properties across the interface, it is found that the interaction forces can be dealt with by introducing fictional dislocations at the image points. These image dislocations will have Burgers vectors that are smaller in magnitude and generally different in orientation from the Burgers vectors of the original dislocations.

Despite this added complexity, it is still possible to exploit a small discrepancy in elastic properties across the grain boundary in order to obtain asymptotic expressions for the dislocation density and for the locations of the furthest and nearest dislocations. In some general pile-up, let $x_{i}$ represent the nondimensionalised distance from the point where the plane of dislocations intersects the grain boundary to the $i$ th dislocation in the pile-up. Then, the equations for a pile-up of dislocations will be of the form

$$
\sum_{j=1, j \neq i}^{n} \frac{1}{x_{i}-x_{j}}+\epsilon \sum_{j=1}^{n} f\left(x_{i}, x_{j}\right)=1, \quad i=1,2, \ldots, n,
$$

where $\epsilon$ is a small parameter representing the change in material properties across the interface and $f\left(x_{i}, x_{j}\right)$ is an order one function representing the stress from an image dislocation at $x_{j}$ that tends to make the dislocation at $x_{i}$ glide. 


\section{Pay 24,2010 \\ Note ArmstrongMonopoles' FinalRey \\ Philosophical Magazine \& Philosophical Magazine Letters}

By the intrinsic symmetry of linear elasticity, we note that

$$
f\left(c x_{i}, c x_{j}\right)=c^{-1} f\left(x_{i}, x_{j}\right)
$$

That is, dilating the entire system by a factor of $c$ will reduce all of the interaction stresses by a factor of $c^{-1}$.

Following [7] and [8], we now consider the asymptotic limit of (22) as $n \rightarrow \infty$. In the outer region away from the boundary layers, we again rescale space by introducing $x=\xi n$. This leads to an integral equation of the form

$$
\int_{0}^{\xi_{0}^{*}} \frac{\rho_{0}\left(\xi^{\prime}\right)}{\xi-\xi^{\prime}}+\epsilon \frac{\rho_{0}\left(\xi^{\prime}\right)}{\xi^{\prime}} k\left(\frac{\xi}{\xi^{\prime}}\right) d \xi^{\prime}=1
$$

where $\xi^{*}$ is the leading order location of the last dislocation, $\rho_{0}(\xi)$ is the leading order dislocation density and

$$
k\left(\frac{\xi}{\xi^{\prime}}\right)=\frac{f\left(\xi, \xi^{\prime}\right)}{\xi^{\prime}}
$$

Note that we are only able to construct $k(t)$ in this way because $f\left(x_{i}, x_{j}\right)$ satisfies (23).

Equation (24) is in the form of the integral equations analysed in [5]. If an appropriate factorisation can be found for

$$
K(s)=\int_{0}^{\infty} k(u) u^{s-1} d u
$$

it is possible to use the Wiener-Hopf method to obtain a closed-form solution for the dislocation density, $\rho_{0}(\xi)$. Even if a Wiener-Hopf factorisation is unworkable, progress might be made by expressing $\rho_{0}(\xi)$ and $\xi_{0}^{*}$ as asymptotic series in powers of $\epsilon$ and seeking a regular perturbation solution to (24).

The next correction in $n$ to the position of the final dislocation can be obtained by proposing a rescaling $\xi=\xi_{0}^{*}+n^{-\frac{2}{3}} \tau$ and following the procedure given in [9] for the boundary layer near the turning point.

In order to find the positions of dislocations in the boundary layer near the grain boundary, it is necessary to determine the appropriate scaling in this region, which (unlike the outer scalings) is sensitive to the form of the interaction with the image dislocations. Following [7], it is possible to proceed using a general inner scaling of the form $x=n^{-\frac{a}{1-a}} \eta$ where $0<a<1$, leaving $a$ to be determined as a function of $\lambda$. More simply, the analogy with [5] indicates that $a$ will be the largest real root of $K(s)$ in $(0,1)$, where $K(s)$ is defined in (25). Even for complicated functions $K(s)$, an approximation to $a(\lambda)$ can be obtained by performing a regular perturbation expansion in powers of $\lambda$ for the root of $K(s)$ near $s=\frac{1}{2}$.

Once the rescaling has been found, the procedure in the boundary layer near the grain boundary will be identical to that described in Sections 2.2 and 3 above. As before, we will find that the location of the first dislocation in a pile-up against a grain boundary will scale like $\lambda n^{-\frac{a}{1-a}}$ as $\lambda \rightarrow 0$ and $n \rightarrow \infty$, while the location of the first dislocation in a pile-up against a lock and a grain boundary will scale like $n^{-\frac{a}{1-a}}$. 


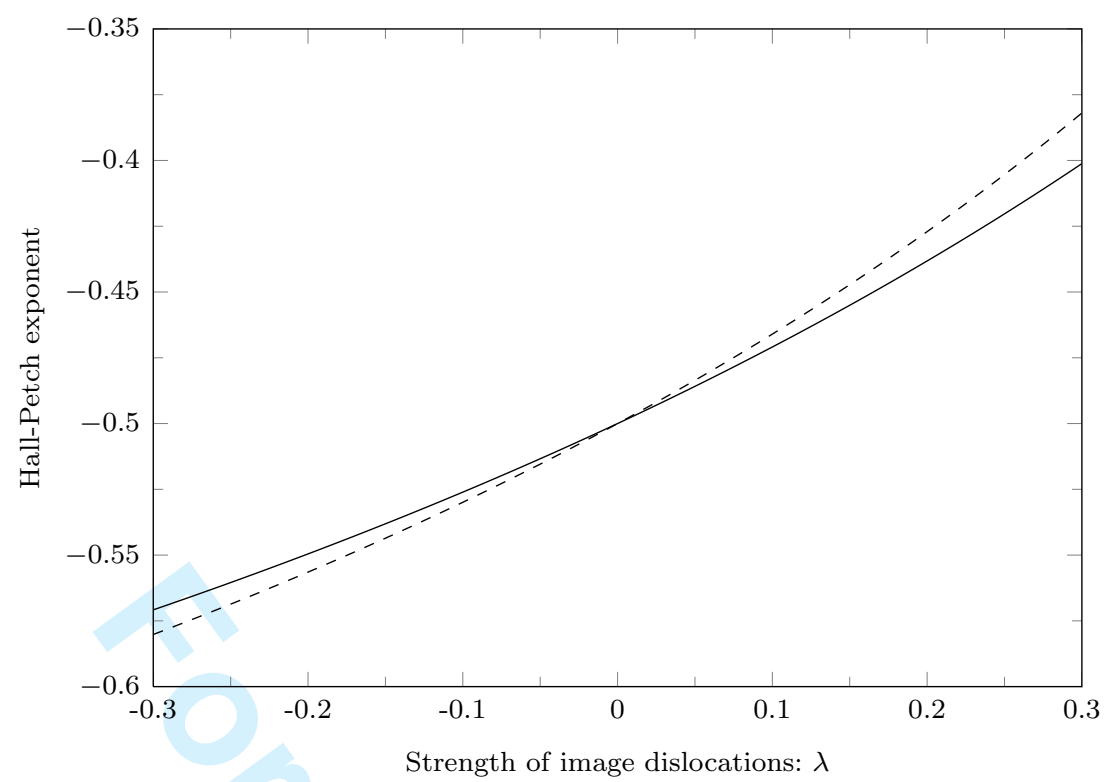

Figure 3. 'Hall-Petch' exponents obtained from the Armstrong-Head formula (26), shown as a solid line, and the asymptotic formula (27), shown as a dashed line. Over the physical range of $\lambda$ values considered, the exponent never deviates far from -0.5 .

\section{Discussion}

\subsection{The Hall-Petch criterion}

Armstrong and Head [1] hypothesised that brittle fracture of a polycrystalline material would first occur when a dislocation becomes locked near a grain boundary and then a second dislocation is pushed to within a Burger's vector length of the locked dislocation. Thus, they considered the stress required to give $\frac{\mu b}{2 \pi \tau} x_{1}=b$ in the problem of a pile-up against a grain boundary and a lock, assuming that the pile-up took place on the scale of a grain so that $x_{n}=l$, where $l$ is the grain size.

This led to the formula

$$
\tau_{\text {crit }}=\frac{\mu}{\pi}\left(\frac{1.9}{2}(1+0.9 \lambda)^{1-1.1 \lambda}\right)^{\frac{1}{2-1.1 \lambda}}\left(\frac{l}{b}\right)^{-\frac{1-1.1 \lambda}{2-1.1 \lambda}}
$$

where $\tau_{\text {crit }}$ is the critical stress for fracture. The equivalent expression based on equations (6) and (20) is

$$
\tau_{\text {crit }}^{(0)}=\frac{\mu}{\pi}\left(\frac{\alpha_{1}^{2}}{16}\left(1+\frac{2 \lambda}{\pi}\right)^{1-\frac{4}{\pi} \lambda}\right)^{\frac{1}{2-\frac{4}{\pi} \lambda}}\left(\frac{l}{b}\right)^{-\frac{1-\frac{4}{\pi} \lambda}{2-\frac{4}{\pi} \lambda}}
$$

Although (5) is more accurate than (6), it is not possible to obtain a closed-form expression for $\tau_{\text {crit }}$ based on (5) and (20).

As in [1], (27) predicts that $\tau_{\text {crit }}$ is proportional to a negative power of $l$ that depends on $\lambda$. Figure (3) shows that the exponents based on (26) and (27) never vary far from $-\frac{1}{2}$ and the two approaches yield results that are always very close to each other. For small $\lambda,(27)$ yields

$$
\tau_{\text {crit }} \propto l^{-\left(\frac{1}{2}-\frac{\lambda}{\pi}\right)}
$$

As noted by Armstrong and Head [1], taking $\lambda=0$ leads to $\tau_{\text {crit }} \propto l^{-\frac{1}{2}}$, which is 


\section{ay $24,2010 \quad 13: 13$
Paqe $\mathbf{1 1}$ of $\mathbf{1 2}$ \\ Philosophical Magazine \& Philosophical Magazine Letters}

a special case of the Hall-Petch criterion:

$$
\tau_{\text {crit }}=\tau_{0}+k l^{-\frac{1}{2}},
$$

where $\tau_{0}$ and $k$ are material-specific constants obtained by experiment.

However, neither (26) nor (27) can account for the $\tau_{0}$ term in (29). Moreover, it is unlikely that taking further terms in the asymptotic expansions for $x_{1}$ and $x_{n}$ would yield a $\tau_{0}$ term. Instead, $\tau_{0}$ needs to be associated with the Peierls friction stress or similar mechanism.

\subsection{Comparison with other work}

It is interesting to note that Armstrong and Head [1] are not the only researchers to use curve fitting in order to approximate the locations of the nearest and furthest dislocations in a pile-up. Contemporary with their work, Chou [2] considered the problem of finding the position of the furthest dislocation when $\lambda=0$ or $\lambda=1$. In [2], as in [9], singular integral equations were used and the results obtained are equivalent. Based on his work, Chou [2] hypothesised a linear relationship between $\xi_{n}$ and $\lambda$ :

$$
\xi_{n}=2+(\pi-2) \lambda, \quad 0 \leq \lambda \leq 1 .
$$

Although this expression fails to incorporate the effects of the boundary layer, we find that it gives results that are very close to the leading order expression in (3) for a range of $\lambda$ values. Equation (6) is, however, a superior approximation to (3) when $\lambda$ is small and the correction term in (5) is important unless $n$ is very large.

Wagoner [10] used numerical techniques to find the equilibrium positions of dislocations in pile-ups against interfaces in anisotropic materials. Although no attempt was made to fit formulae for the locations of the furthest and nearest dislocations, the position of the dislocation nearest to the interface was estimated by balancing the forces from the real dislocations in the pile-up with the force from the nearest image dislocation. This is analogous to the method described in Section 2.2, but [10] found a surprisingly poor agreement between estimated position and calculated position. The main reason for this is that [10] did not have the correct scaling for the dependence of $x_{1}$ on $n$. Thus, insights from asymptotic analysis can be used to obtain results that are not immediately intuitive.

\section{Conclusions}

We have used the asymptotic methods of Voskoboinikov et al. [8] to obtain expressions for the locations of the first and last dislocations in a pile-up against a grain boundary and these expressions have been compared with numerical results. Our asymptotic expressions have the same form as the formulae obtained by Armstrong and Head [1] using curve fitting, but some of the parameters are different. As expected, the asymptotic expressions are better than the fitted formulae when $n$ is large and $\lambda$ is small.

For a pile-up against a grain boundary where the mismatch (and hence $\lambda$ ) is small, we recovered the same system of equations in the inner region as for a pile-up against a locked dislocation. This can be explained by observing that the first dislocation becomes very close to the grain boundary as $\lambda \rightarrow 0$ and the other dislocations begin to feel it as if it were locked at the grain boundary. Thus, pile-ups 


\section{Philosophical Magazine \& Philosophical Magazine Letters}

against grain boundaries may lead to situations that are physically equivalent to pile-ups against locks. This is true both for the simple pile-ups of screw dislocations analysed in detail and for more complicated configurations.

\section{Acknowledgements}

This publication is based on work supported by Award No. KUK-C1-013-04, made by King Abdullah University of Science and Technology (KAUST).

I would like to acknowledge the contributions of John R. Ockendon (Oxford), S. Jonathan Chapman (Oxford), Roman E. Voskoboynikov (Kurchatov) and Graeme C. Wake (Massey), with whom I discussed various aspects of this work.

\section{References}

[1] R. W. Armstrong and A. K. Head. Dislocation queueing and fracture in an elastically aniostropic material. Acta Metallurgica, 13(7):759-764, 1965.

[2] Y. T. Chou. Linear dislocation arrays in heterogeneous materials. Acta Metallurgica, 13(7):779 - 783, 1965.

[3] J. D. Eshelby, F. C. Frank, and F. R. N. Nabarro. The equilibrium of linear arrays of dislocations. Philosophical Magazine Series 7, 42(327):351-364, 1951.

[4] A. K. Head. Edge dislocations in inhomogeneous media. Proceedings of the Physical Society B, 66(9):793-801, 1953.

[5] J. Kuang and T. Mura. Dislocation pile-up in two-phase materials. Journal of Applied Physics, 39(1):109-120, 1968.

[6] M. O. Tucker. Plane boundaries and straight dislocations in elastically anisotropic materials. Philosophical Magazine, 19(162):1141-1159, 1969.

[7] R. E. Voskoboinikov, S. J. Chapman, J. B. Mcleod, and J. R. Ockendon. Asymptotics of edge dislocation pile-up against a bimetallic interface. Mathematics and Mechanics of Solids, 14(1-2):284 $-295,2009$.

[8] R. E. Voskoboinikov, S. J. Chapman, and J. R. Ockendon. Continuum and discrete models of dislocation pile-ups. II. Pile-up of screw dislocations at the interface in a bimetallic solid. Philosophical Magazine Letters, 87(9):669-676, 2007.

[9] R. E. Voskoboinikov, S. J. Chapman, J. R. Ockendon, and D. J. Allwright. Continuum and discrete models of dislocation pile-ups. I. Pile-up at a lock. Journal of the Mechanics and Physics of Solids, 55(9):2007 - 2025, 2007.

[10] R. H. Wagoner. Calculating dislocation spacings in pile-ups at grain boundaries. Metallurgical Transactions A, 12A:2015-2023, 1981. 